\title{
Induction of osteoclast formation by LOX mutant (LOXG473A) through regulation of autophagy
}

\author{
Bo Zhang $^{1 \#}$, Chenglin Luo ${ }^{2 \#}$, Wenjin Xiao ${ }^{3}$ \\ ${ }^{1}$ Department of Radiology, The Second Affiliated Hospital of Soochow University, Suzhou, China; ${ }^{2}$ Department of Gastroenterology, Suzhou \\ Xiangcheng People's Hospital, Suzhou, China; ${ }^{3}$ Department of Endocrinology, Second Affiliated Hospital of Soochow University, Suzhou, China \\ Contributions: (I) Conception and design: W Xiao; (II) Administrative support: W Xiao; (III) Provision of study materials or patients: B Zhang, W \\ Xiao; (IV) Collection and assembly of data: B Zhang, C Luo; (V) Data analysis and interpretation: C Luo; (VI) Manuscript writing: All authors; (VII) \\ Final approval of manuscript: All authors. \\ "These authors contributed equally to this work. \\ Correspondence to: Professor Wenjin Xiao. Department of Endocrinology, The Second Affiliated Hospital of Soochow University, 1055 Sanxiang \\ Road, Suzhou 215004, China. Email: xwj19840507@163.com.
}

Background: Lysyl oxidase (LOX) has been identified to modulate osteoclast activity, so we explored the role of $\mathrm{LOX}_{\mathrm{G} 473 \mathrm{~A}}$, the highest frequency single nucleotide polymorphism in LOX, in osteoclast formation and its potential relationship to autophagy.

Methods: The ability of the LOX mutant, $\operatorname{LOX}_{\mathrm{G} 473 \mathrm{~A}}$, to promote autophagy and osteoclast formation was evaluated in the pre-osteoclast cell line RAW264.7. Furthermore, autophagy-related protein expression and autophagosomes were detected by western blot and electron microscopy, respectively. Simultaneously, osteoclast formation and resorption ability were also detected using TRAP staining assay and bone resorption assay. In addition, the osteoclast-related proteins and mRNAs, as well as p-AMPK $\alpha$ and p-mTOR proteins, were further evaluated by western blot and qPCR assays.

Results: Autophagy inhibitor 3-MA suppressed the Beclin-1 and ATG5 protein levels and the ratio of LC3-II to LC3-I, as well as autophagosome formation in RAW264.7 transfected with the MUT plasmid and enhanced p62 protein expression. Simultaneously, 3-MA also reduced osteoclast formation and resorption, as well as the F-actin ring level of osteoclasts. In addition, 3-MA inhibited osteoclast-related protein and mRNA expression, including NFATC1, ACP5, CTSK. And the autophagy-related pathway protein p-AMPK $\alpha$ was increased and p-mTOR was reduced by 3-MA treatment. However, autophagy agonist RAPA reversed the effect of 3-MA on RAW264.7 with $\mathrm{LOX}_{\mathrm{G} 473 \mathrm{~A}}$ mutation, indicating that promoting autophagy could enhance the ability of $\operatorname{LOX}_{\mathrm{G} 473 \mathrm{~A}}$ to induce osteoclast formation.

Conclusions: LOX mutant $\left(\operatorname{LOX}_{\mathrm{G} 473 \mathrm{~A}}\right)$ might promote osteoclast formation for RAW264.7 by enhancing autophagy via the AMPK/mTOR pathway, which is a new direction for bone disease research.

Keywords: Autophagy; lysyl oxidase (LOX); LOXG473A; osteoclast formation; RAW264.7

Submitted Jul 30, 2021. Accepted for publication Sep 18, 2021.

doi: 10.21037/atm-21-4474

View this article at: https://dx.doi.org/10.21037/atm-21-4474

\section{Introduction}

Bone is a tissue that needs continuous self-renewal to ensure the integrity of the skeleton (1). Bone resorption and formation is a dynamic balanced process; once the balance is disturbed, it will lead to bone-related diseases such as osteoporosis, osteoarthritis, and osteosarcoma $(2,3)$. Of these, osteoporosis, with its features of low bone thickness and high danger of fracture, is a public health issue $(4,5)$. Osteoporosis-related fracture is a common cause of injury and death in the elderly (6). Osteoclasts, which originate 
from precursor cells of macrophages/monocytes, play a key role in bone resorption (4) and abnormal formation of osteoclasts is considered to be the main cause of osteoporosis. In osteoporosis, the formation of osteoblasts increased (7), which means inhibition the activity of osteoclasts may alleviate osteoporosis.

Osteoclast differentiation and formation is a complicated biological procedure involving a variety of signaling pathways and cytokines, among which, receptor activator of nuclear factor kappa B ligand (RANKL) and macrophage colony stimulating factor (M-CSF) are the foremost cytokines (8). M-CSF is an important factor for the proliferation of osteoclast precursor cells, while RANKL stimulates osteoclast precursor cells to mature into osteoclasts (9). Moreover, early studies have shown that extracellular lysyl oxidase (LOX) can catalyze the oxidative deamination of amino acid residues in collagen (10), and the aldehydes produced can form important cross-links to stabilize functional collagen, such as bone collagen (11). Independent of the RANKL system, LOX can also induce bone metastasis and bone destruction in breast cancer patients (12). Human $\mathrm{CD} 14^{+}$monocytes cultured without RANKL but with LOX form osteoclasts (13), indicating that LOX might be a new regulator of osteoclast activation.

G473A is situated in the highly conserved area of the LOX gene and is also currently the most widely studied mutation site, where arginine at position 158 of the encoded protein can be mutated to glutamine, thereby inhibiting the degradation of the LOX proenzyme to the LOX propeptide and thus increasing the expression of active LOX (14). In addition, a LOX mutant $\left(\operatorname{LOX}_{\mathrm{G} 473 \mathrm{~A}}\right)$ is also related to abnormalities in various diseases $(15,16)$. Therefore, $\operatorname{LOX}_{\mathrm{G} 473 \mathrm{~A}}$ is an important gene locus that affects human health. Recently, we confirmed for the first time that $\operatorname{LOX}_{\mathrm{G} 473 \mathrm{~A}}$ is closely related to lumbar fractures in postmenopausal women (17). Compared with the GG genotype, the fracture risk of people with GA and AA genotypes increased by 1.28- and 1.74-fold, respectively (17). Nevertheless, the mechanism by which $\mathrm{LOX}_{\mathrm{G} 473 \mathrm{~A}}$ drives osteoclast differentiation remains vague in osteoporosis.

Autophagy can degrade lipids, damaged proteins and organelles to maintain cell homeostasis, and it is also an evolutionary conservative process $(18,19)$. Autophagy is a pleiotropic function, involving cell survival, nutritional supply during hunger, antigen presentation, and resistance to pathogens (20). Research in recent years has shown that autophagy participates in the degradation of osteocytes, osteoclasts, and osteoblasts, which may be a novel pathogenic mechanism for bone homeostasis (21). Research has shown that kaempferol restrains bone resorption and osteoclast differentiation by inhibiting autophagy (20). Based on the previously known involvement of $\mathrm{LOX}_{\mathrm{G} 473 \mathrm{~A}}$ in the regulation of osteoclast formation (17), and the role of autophagy in the regulation of osteoclast formation (20), it is not clear whether they have a reciprocal regulatory relationship. In this study, the pre-osteoclast cell line RAW264.7 were used to explore the role of $\operatorname{LOX}_{\mathrm{G} 473 \mathrm{~A}}$ in osteoclast differentiation and the potential role of autophagy in osteoclast differentiation, giving insight to the treatment of osteoporosis. We present the following article in accordance with the MDAR reporting checklist (available at https://dx.doi.org/10.21037/atm-21-4474).

\section{Methods}

\section{Cell culture}

The murine monocytic macrophage cell line RAW264.7 was purchased from the American Type Culture Collection (TIB-71, ATCC USA) and were cultured in $\alpha$-MEM medium (Gibco, USA) with the addition of $60 \mathrm{ng} / \mathrm{mL}$ recombinant mouse RANKL (PeproTech, USA), $10 \mathrm{ng} / \mathrm{mL} \mathrm{M-CSF}$ (R\&D Systems, USA) and $10 \% \mathrm{FBS}$ for 5 days at $37{ }^{\circ} \mathrm{C}$ in $5 \% \mathrm{CO}_{2}$ atmosphere for osteoclast differentiation (22).

\section{Transfection}

Wild-type LOX (WT) and mutant $\mathrm{LOX}_{\mathrm{G} 473 \mathrm{~A}}$ (MUT) genes were obtained from GenePharma (China). Sequences are shown in Table 1. The WT and MUT genes were cloned into a pcDNA3.1 plasmid. Synthetic plasmids and empty vector (Vector) were transfected into RAW264.7 using the Lipofectamine TM 3000 transfection reagent (Thermo Fisher Scientific, USA) according to the manufacturer's protocol.

\section{TRAP staining assay}

TRAP a marker enzyme of osteoclasts. Different RAW264.7 transfected plasmids were cultured in $\alpha$-MEM medium with the addition of $60 \mathrm{ng} / \mathrm{mL}$ recombinant mouse RANKL, $10 \mathrm{ng} / \mathrm{mL} \mathrm{M}-\mathrm{CSF}$, and 10\% FBS for osteoclast differentiation for 5 days (23). The cells were then simultaneously treated with RAPA or 3-MA. The cells were fixed and stained for TRAP 5b using a TRAP staining kit (Sigma-Aldrich, USA) and the manufacturer's protocol (24). Cells stained red were 
Table 1 Coding sequence (CDS) of LOX gene

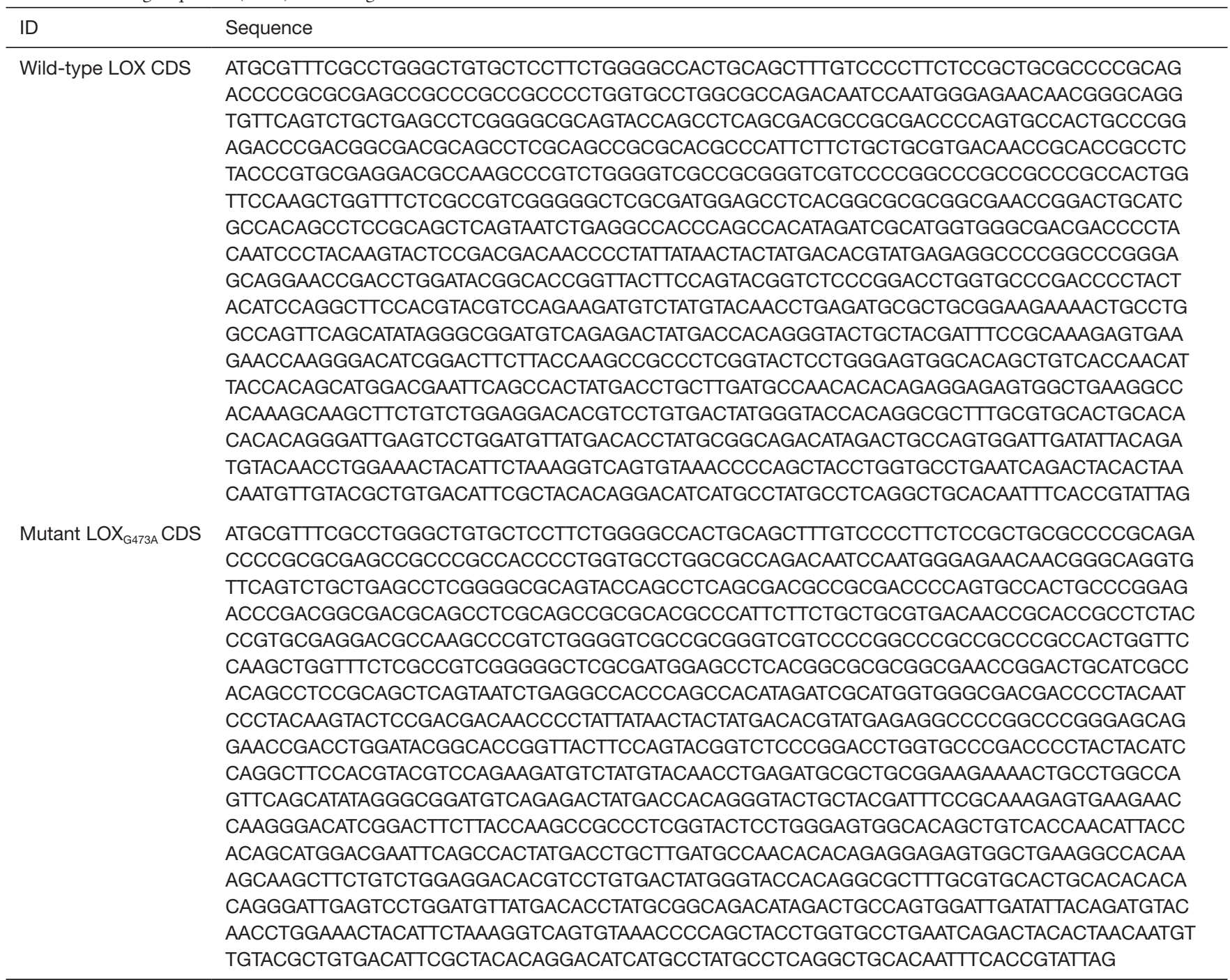

defined as differentiated osteoclast-like cells. Cells with more than 3 nuclei were considered multinucleated cells. The number of TRACP+ cells was counted using ImagePro Plus software.

\section{Bone resorption assay}

Different RAW264.7 transfected plasmids $\left(1.5 \times 10^{3}\right.$ cells/ well) were seeded onto a bone biomimetic synthetic surface (Osteo Assay Surface 24-Well Multiple Well Plates; Corning, USA) (24). The medium was changed every 2 days and after 5 days, the plates were washed with phosphate-buffered saline (PBS) and air-dried for $4 \mathrm{~h}$. The area of osteoclastic absorption was observed under a light microscope and quantified by Image-Pro Plus software.

\section{F-actin ring-formation assay}

The F-actin ring is a precondition for osteoclast bone resorption and the most significant feature of mature osteoclasts. The culture method of RAW264.7 for osteoclastic differentiation in the TRAP staining experiment was used in the ring-formation experiment. RAW264.7 cells were cultured for osteoclast differentiation for 5 days and then fixed with $4 \%$ paraformaldehyde at $4{ }^{\circ} \mathrm{C}$ for $10 \mathrm{~min}$ and stained with $0.1 \%$ phalloidin after the osteoclasts formed (25). The images were observed by confocal microscopy (Leica, Germany). 
Table 2 Primer sequences for quantitative real-time PCR (qPCR) detection

\begin{tabular}{llc}
\hline ID & Sequence (5'-3') & Product length (bp) \\
\hline$\beta$-actin. Forward & CATTGCTGACAGGATGCAGA & 139 \\
$\beta$-actin. Reverse & CTGCTGGAAGGTGGACAGTGA & 117 \\
LOX. Forward & TCTTCTGCTGCGTGACAACC & \\
LOX. Reverse & GAGAAACCAGCTTGGAACCAG & 97 \\
NFATC1. Forward & GACCCGGAGTTCGACTTCG & 118 \\
NFATC1. Reverse & TGACACTAGGGGACACATAACTG & \\
ACP5. Forward & CACTCCCACCCTGAGATTTG & 102 \\
ACP5. Reverse & CATCGTCTGCACGGTTCTG & \\
CTSK. Forward & GAAGAAGACTCACCAGAAGCAG & \\
CTSK. Reverse & TCCAGGTTATGGGCAGAGATT & \\
\hline
\end{tabular}

\section{Electron microscopy}

RAW264.7 transfected with MUT plasmid after 3-MA or RAPA treatment were fixed with $4 \%$ paraformaldehyde for $30 \mathrm{~min}$. Cells were incubated using $1.5 \%$ osmium tetroxide, followed by dehydration with acetone and then they were embedded in Durcupan resin. The prepared sections were stained with lead citrate and observed under TECNAI 10 electron microscope (Philips, Holland) at $60 \mathrm{kV}$ to observe the formation of autophagy bodies.

\section{$q P C R$}

Total RNA from plasmids transfected RAW264.7 cells or 3-MA or RAPA treated cells was separated using the Trizol reagent and cDNA was obtained from mRNA using the PrimeScipt MMLV RT reagent Kit (TakaRa, Japan) based on the manufacturer's protocol. The quantitative real-time PCR (qPCR) reaction was executed using SYBR green (Bio$\mathrm{Rad}, \mathrm{USA})$ as the fluorescent reporter and an ABI-7500. A total of 40 cycles $\left(95^{\circ} \mathrm{C}\right.$ for $30 \mathrm{~s}, 60^{\circ} \mathrm{C}$ for $30 \mathrm{~s}$, and $72{ }^{\circ} \mathrm{C}$ for $2 \mathrm{~min}$ ) were run for each primer. $\beta$-actin was used as a standardized internal control. Primers are shown in Table 2.

\section{Western blot}

Treated cells were dissolved in RIPA buffer including protease inhibitors, and then $20 \mu \mathrm{g}$ protein samples were added to SDS-PAGE gel electrophoresis followed by a transfer of protein to a PVDF membrane. Membranes were sealed using $5 \%$ fat-free milk for $1 \mathrm{~h}$ and then cultured with antibodies against LOX (Abcam, ab174316), Beclin-1 (Abcam, ab207612), LC3B (Abcam, ab192890), p62 (Abcam, ab109012), ATG5 (Abcam, ab108327), mTOR (Abcam, ab134903), p-mTOR (Abcam, ab109268), AMPK $\alpha$ (Abcam, ab32047), p-AMPKa (Abcam, ab133448), NFATC1 (Abcam, ab25916), ACP5 (Abcam, ab238913), CTSK (Abcam, ab239506), and GAPDH (Cell Signaling Technology, \#5174) overnight at $4{ }^{\circ} \mathrm{C}$. After a 30 -min washing step in TBS containing $0.1 \%$ Tween 20 , the membranes were cultured with HRP (horseradish peroxidase)-conjugated secondary antibodies (Abcam, ab205718 and ab6728). Visualization was carried out using Pierce ${ }^{\mathrm{TM}} \mathrm{ECL}$ western blotting substrate (Thermo Fisher Scientific, USA) according to the manufacturer's protocol. Analysis was performed with Image-Pro Plus software.

\section{Statistical analysis}

All experiments were performed thrice. All data were statistically analyzed with Graphpad Prism 8.0.2, using one-way analysis of variation, followed by Tukey's multiple comparisons test. $\mathrm{P}<0.05$ were considered statistically significant.

\section{Results}

Effect of LOX ${ }_{G 473 A}$ on level of autophagy in $R A W 264.7$

We constructed WT and MUT LOX plasmids. Simultaneously, the MUT and Vector were transfected into RAW264.7. The sequencing results showed that the 
$\mathrm{G}$ in $L O X$ was successfully mutated to A (Figure $1 A$ ). In addition, the mRNA and protein of LOX in the WT and MUT transfected groups were significantly higher than in the Vector transfected group, suggesting that the WT and MUT plasmids were successfully transfected into RAW264.7 (Figure 1B-1D). The western blot analysis results also implied that $\mathrm{LOX}$ and $\mathrm{LOX}_{\mathrm{G} 473 \mathrm{~A}}$ promoted the levels of Beclin-1, ATG5, and LC3-II protein, as well as the conversion of LC3-II to LC3-I, and suppression of LC3-I and p62 protein expression, suggesting that both $\mathrm{WT}$ and MUT induced autophagy (Figure 1B,1E-1f). Moreover, the Beclin-1, ATG5, and LC3-II protein expressions in the MUT group were higher than in the WT group (Figure 1B, $1 E, 1 G, 17)$. The LC3-I and p62 protein expressions in the MUT group were lower than those in the WT group, showing that $\operatorname{LOX}_{\mathrm{G} 473 \mathrm{~A}}$ was better able to induce cell autophagy than LOX (Figure 1B,1F,1I).

\section{Effect of $L O X_{G 473 A}$ on osteoclast formation in $R A W 264.7$}

We detected the role of $\mathrm{LOX}_{\mathrm{G} 473 \mathrm{~A}}$ in the bone formation and bone resorption of RAW264.7 cells differentiated into osteoclasts. TRAP staining showed significantly increased osteoclast formation in the WT group compared with the Vector group, and significantly increased osteoclast formation in the MUT group compared with the WT group (Figure $2 A$ ). In addition, bone resorption experiments indicated that the bone resorption area was much greater in the WT group compared with the Vector group, and remarkably higher in the MUT group compared with the WT group (Figure 2B), suggesting that MUT promoted bone resorption by osteoclasts.

\section{Effect on $L O X_{G 473 A}$ promotion of autophagy by 3-MA or RAPA}

Western blot analysis indicated that in the MUT + 3-MA group, the p62 protein expression was remarkably higher than in the MUT group, but the Beclin-1 and ATG5 protein levels, as well as the conversion of LC3-I to LC3-II, were remarkably lower than in the MUT group (Figure $3 A, 3 B$ ). RAPA showed the opposite effects on the expression levels of the above proteins, indicating that RAPA enhanced the ability of $\mathrm{LOX}_{\mathrm{G} 473 \mathrm{~A}}$ to promote autophagy and 3-MA reduced the ability of $\mathrm{LOX}_{\mathrm{G} 473 \mathrm{~A}}$ to promote autophagy (Figure $3 A, 3 B$ ). Furthermore, the electron microscopy results also showed that 3-MA suppressed the production of autophagosomes in RAW264.7 cells transfected with
MUT plasmids, and RAPA promoted the production of autophagosomes inRAW264.7 transfected with MUT plasmids (Figure 3C).

\section{Effect on $\mathrm{LOX}_{G 473 A}$ induction of osteoclast formation by 3-MA or RAPA}

Our results showed that RAPA enhanced the ability of $\mathrm{LOX}_{\mathrm{G} 473 \mathrm{~A}}$ to promote autophagy and 3-MA reduced the ability of $\operatorname{LOX}_{\mathrm{G} 473 \mathrm{~A}}$ to promote autophagy. Furthermore, the effect of 3-MA or RAPA on the ability ofLOX $\mathrm{G}_{\mathrm{G} 473 \mathrm{~A}}$ to induce osteoclastogenesis was further explored. The TRAP staining results showed that in the MUT + 3-MA group, the number of red cells was remarkably lower in contrast to the MUT group, while in the MUT + RAPA group, the number was much higher in contrast to the MUT group (Figure 4A). The result of semiquantitative analysis also indicated that the number of osteoclasts in the MUT + 3-MA group was much fewer than in the MUT group and the number of osteoclasts in the MUT + RAPA group was much greater than in the MUT group (Figure 4A). The results indicated that 3-MA reduced osteoclast differentiation of RAW267.4 and RAPA enhanced osteoclast differentiation of RAW267.4 (Figure 4A). In addition, bone resorption experiments showed that in the MUT + 3-MA group, the area of bone resorption was markedly less than in the MUT group, while in the MUT + RAPA group, the area of the cells was markedly higher in contrast of the MUT group (Figure 4B), suggesting that 3-MA inhibited osteoclast resorption and RAPA promoted osteoclast resorption.

\section{Effect of blocking autophagy on F-actin expression in osteoclasts}

We also tested whether 3-MA and RAPA could affect the production of the F-actin rings of osteoclasts (26). Our results showed that 3-MA inhibited the number of $\mathrm{F}$-actin rings forming in RAW267.4 cells induced by M-CSF $(10 \mathrm{ng} / \mathrm{mL})$ and recombinant mouse RANKL $(60 \mathrm{ng} / \mathrm{mL})$ (Figure $5 A, 5 B$ ). RAPA reversed this phenomenon (Figure $5 A$, $5 B)$. These results indicated that 3-MA might promote osteoclast differentiation by blocking autophagy, while RAPA might induce osteoclast differentiation by promoting autophagy.

\section{Effect of AMPK/p-mTOR in osteoclast differentiation}

In order to explore if 3-MA and RAPA affect the 

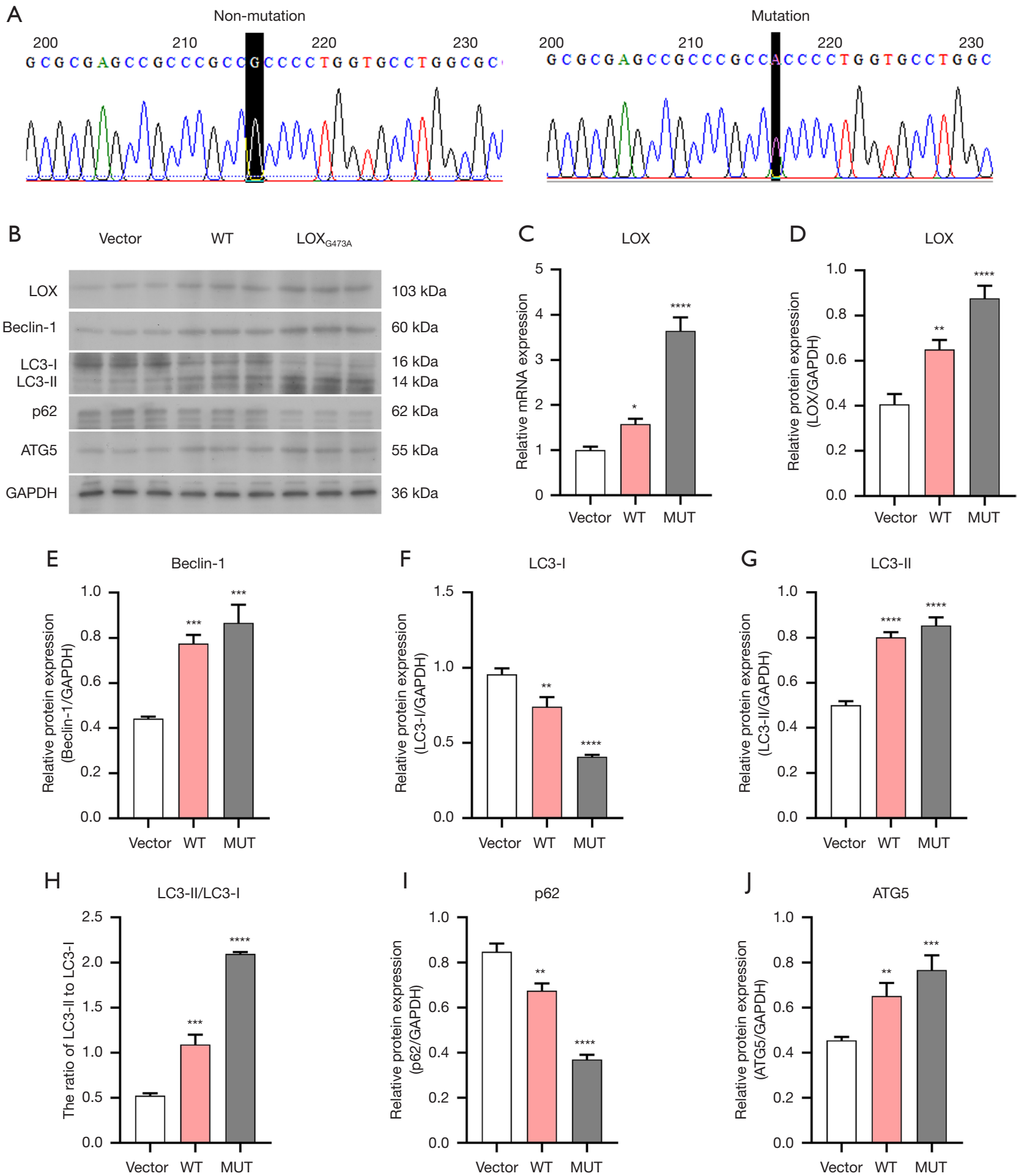

Figure 1 Effect of LOX mutant $\left(\operatorname{LOX}_{\mathrm{G} 473 \mathrm{~A}}\right)$ on autophagy level in RAW264.7 cells. (A) Mutation site of LOX gene. (B) The LOX protein and autophagy-related protein expressions in RAW264.7 transfected with wild-type LOX (WT) and mutant LOX $_{\mathrm{G} 473 \mathrm{~A}}$ (MUT) plasmids tested by western blot. (C) LOX mRNA expression in RAW264.7 transfected with WT and MUT plasmids tested by qPCR. (D-J) Protein expression level in (B) quantified using Image-Pro Plus software. *, $\mathrm{P}<0.05$; **, $\mathrm{P}<0.01$; ${ }^{* * *}, \mathrm{P}<0.001$; and ${ }^{* * * *}, \mathrm{P}<0.0001$ vs. Vector represent significant differences. 
A
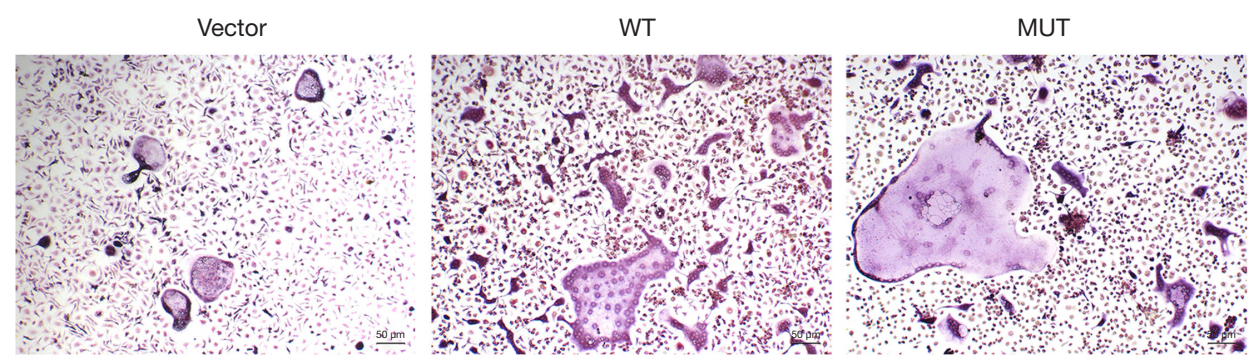

B
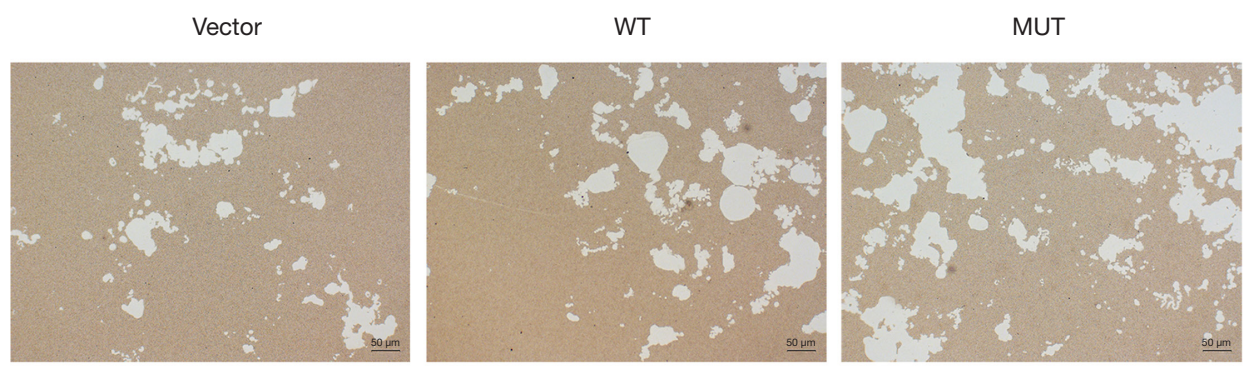
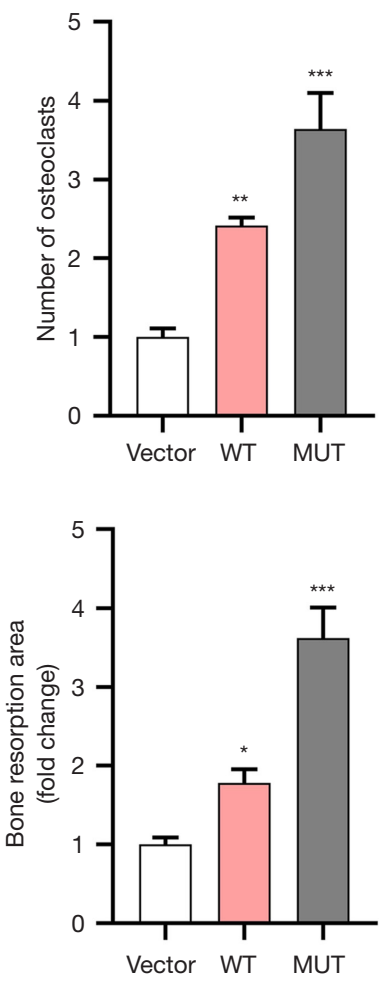

Figure 2 Effect of LOX mutant $\left(\operatorname{LOX}_{\mathrm{G} 473 \mathrm{~A}}\right)$ on the differentiation of RAW264.7 into osteoclasts. (A) Osteoclast expression in RAW264.7 transfected with wild-type LOX (WT) and mutant $\operatorname{LOX}_{\mathrm{G} 473 \mathrm{~A}}$ (MUT) plasmids detected by TRAP staining. Magnification $\times 100$. (B) Level of osteoclast resorption level in RAW264.7 transfected with WT and MUT plasmids analyzed by Image-Pro Plus software. *, P<0.05; **, $\mathrm{P}<0.01$ vs. Vector and ***, $\mathrm{P}<0.001$ vs. WT represent significant differences.

differentiation of osteoclasts by blocking autophagy and the underlying mechanism of their effects, western blot and $\mathrm{qPCR}$ assays were carried out to detect the levels of related proteins and mRNAs. The results implied that 3-MA inhibited the NFATC1, ACP5 and CTSK protein expressions in the MUT group and that RAPA promoted the NFATC1, ACP5 and CTSK protein expressions in MUT group (Figure 6A,6B). Simultaneously, 3-MA also inhibited the NFATC1, CTSK and ACP5 mRNA levels in the MUT group, and RAPA also promoted the NFATC1, CTSK and ACP 5 mRNA levels in the MUT group (Figure 6C). These results further illustrated that blocking autophagy could inhibit the osteoclast differentiation ability of RAW264.7 transfected with MUT plasmids, and that promoting autophagy could reverse this phenomenon. Further analysis showed that 3-MA could inhibit the protein levels of p-AMPK $\alpha$ and promote the protein levels of p-mTOR (Figure $6 A, 6 B$ ), that RAPA had the opposite effect, suggesting that AMPK $\alpha / \mathrm{p}-\mathrm{mTOR}$ is involved in autophagy-affected osteoclast differentiation.

\section{Discussion}

Osteoporosis is a common bone metabolism disease characterized by bone loss, bone microstructural changes and increased bone fragility (27). Excessive activation of osteoclasts leads to bone metabolism illness such as rheumatoid arthritis and osteoporosis $(28,29)$. Studies have shown that inhibiting the differentiation of osteoclasts and reducing the excessive activation of osteoclasts is an effective therapy for bone loss diseases (30). In addition, osteoporosis is also a polygenic disease, although most of the genes related to osteoporosis are still unknown (31). In this study, the pre-osteoclast cell line RAW264.7 was used to study the role of the LOX mutant, $\operatorname{LOX}_{\mathrm{G} 473 \mathrm{~A}}$, in osteoclast differentiation. $\operatorname{LOX}_{\mathrm{G} 473 \mathrm{~A}}$ showed a significant increase in LOX expression, as well as osteoclast formation and autophagy in RAW264.7. Further studies showed that this phenomenon could be inhibited by 3-MA and enhanced by RAPA, suggesting that $\mathrm{LOX}_{\mathrm{G} 473 \mathrm{~A}}$ can induce excessive activation of osteoclasts via regulation of autophagy. 

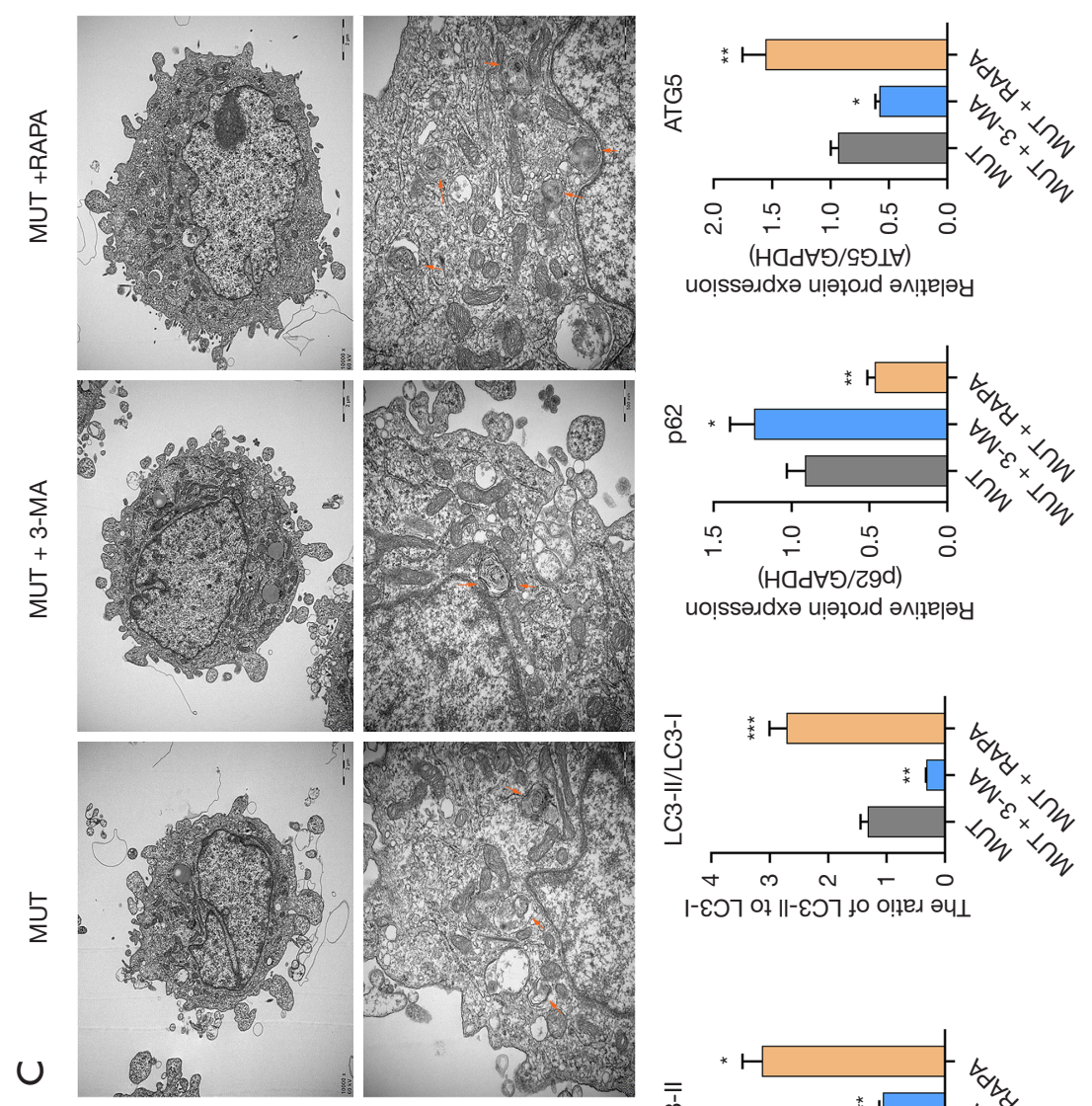

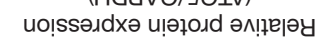

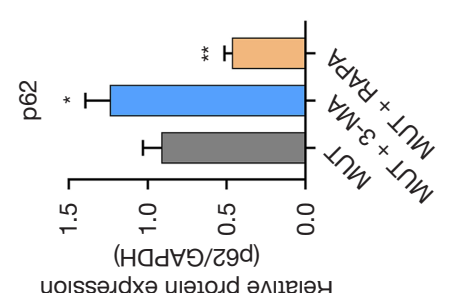

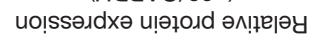

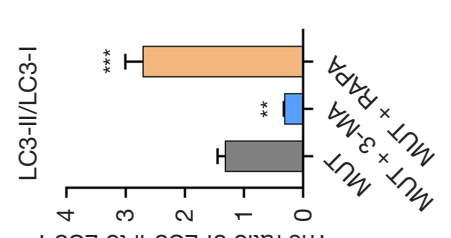

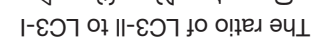

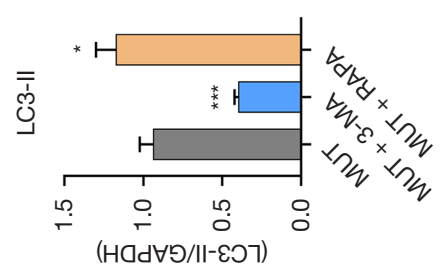

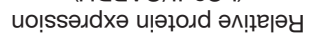
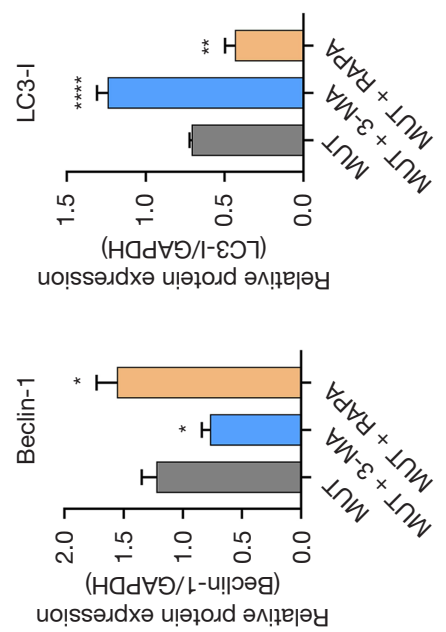

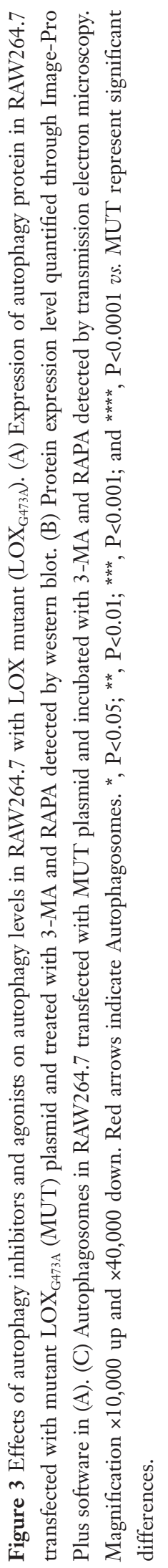


A
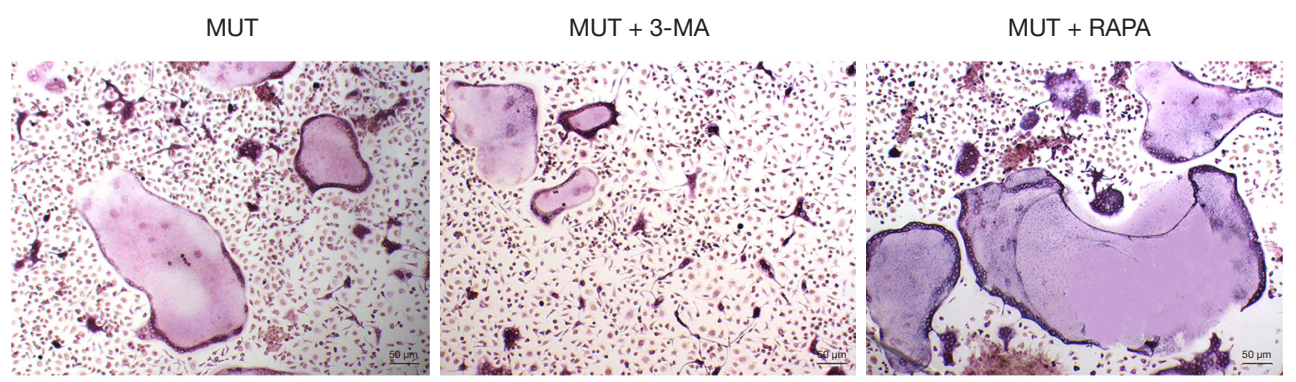

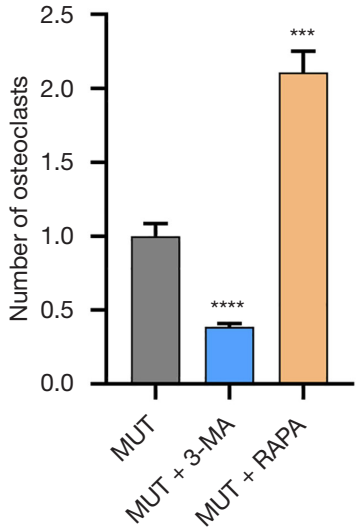

B
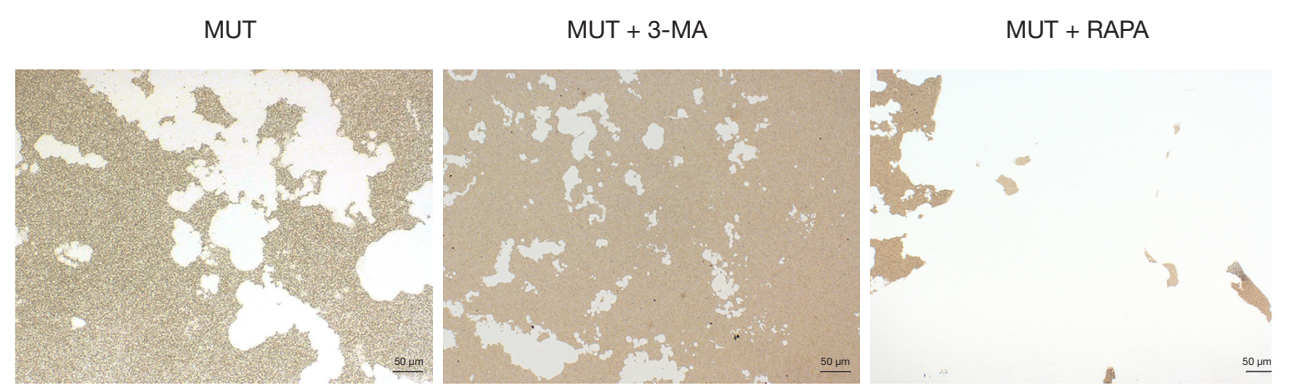

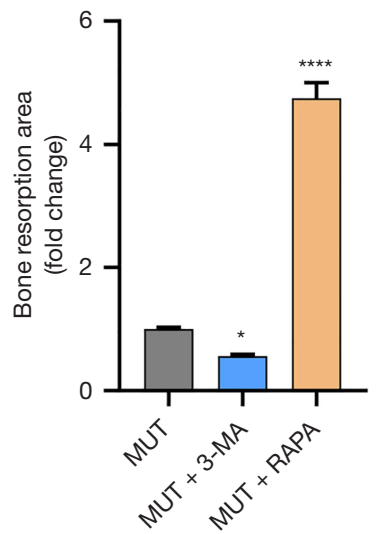

Figure 4 Effects of autophagy inhibitors and agonists on osteoclast differentiation and resorption. Magnification $\times 100$. (A) Osteoclast expression in RAW264.7 transfected with mutant LOX $_{\mathrm{G} 473 \mathrm{~A}}$ (MUT) plasmid and treated with 3-MA and RAPA detected by TRAP staining. Semiquantitative analysis of the number of osteoclasts by Image-Pro Plus software. (B) Level of osteoclast resorption in RAW264.7 transfected with MUT plasmid and treated with 3-MA and RAPA analyzed by Image-Pro Plus software. ${ }^{*}, \mathrm{P}<0.05 ;{ }^{* * *}, \mathrm{P}<0.001 ;$ and ${ }^{* * * *}$, $\mathrm{P}<0.0001$ vs. MUT represents significances.

Studies have shown that LOX and interleukin (IL)6 synergistically induced osteoclast differentiation of RANKL-dependent mouse primary bone marrow cells (32). Human $\mathrm{CD} 14^{+}$monocytes treated with LOX can differentiate into osteoclasts (13). Interestingly, our research also found that in the WT group, both osteoclast number and bone resorption area of processed RAW264.7 were remarkably higher compared with the Vector group. That result further illustrated that LOX can induce osteoclasts. In addition, studies show that $\mathrm{LOX}_{\mathrm{G} 473 \mathrm{~A}}$ is associated with diabetic foot ulcers, compared with healthy individuals (33). Our results indicated that $\mathrm{LOX}_{\mathrm{G} 473 \mathrm{~A}}$ enhanced osteoclast formation inRAW264.7 according to TRAP staining, compared with the WT group, showing that $\operatorname{LOX}_{\mathrm{G} 473 \mathrm{~A}}$ might be related to the activation of osteoclasts and a correlation between LOX mutation and osteoporosis.

Interestingly, we found the osteoclast formation and resorption, as well as $\mathrm{F}$-actin ring production of osteoclasts, were inhibited in RAW264.7 with the $\operatorname{LOX}_{G 473 \mathrm{~A}}$ mutation through suppression of the expression of autophagosomes and autophagy-related proteins. Other research has also shown that kaempferol inhibits the expression of NFATC1, and also the levels of autophagy-related proteins, including P62 and Beclin-1, as well as the ratio of LC3-I/LC3-II in RAW264.7 (20). The expression of TRAP $^{+}$multinucleated cells and TRAP mRNA were also downregulated in bone marrow macrophages induced by IL-17A via inhibition of autophagy (34). These findings show that inhibiting autophagy could alleviate $\mathrm{LOX}_{\mathrm{G} 473 \mathrm{~A}}$-induced osteoporosis. However, our study also demonstrated that $\mathrm{LOX}_{\mathrm{G} 473 \mathrm{~A}}$ can 
A
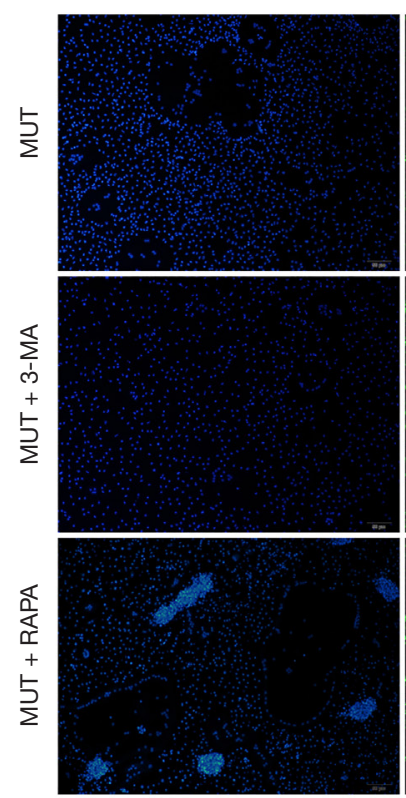

F-actin
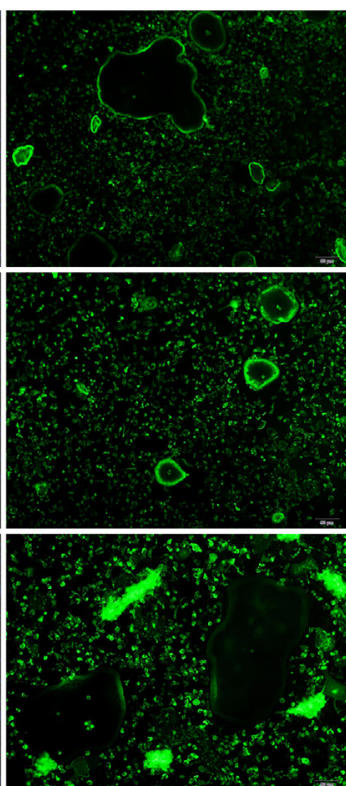

Merge

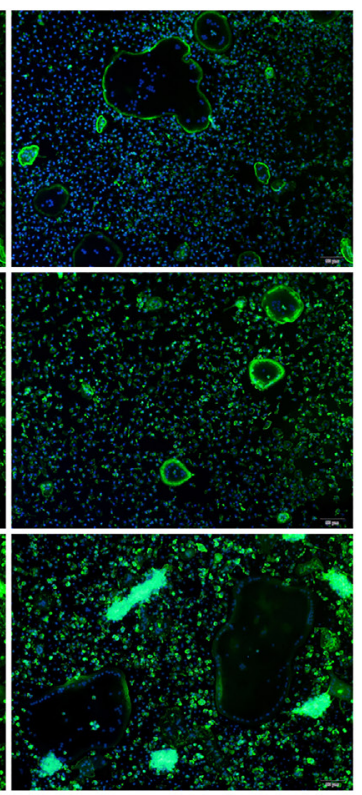

B

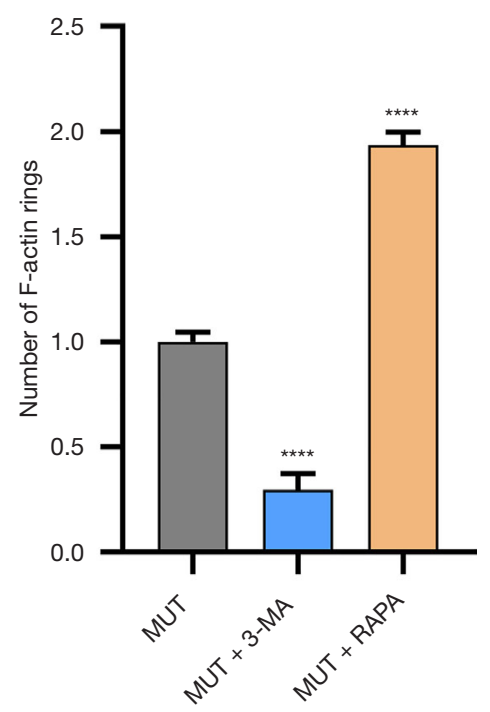

Figure 5 Effects of autophagy inhibitors and agonists on F-actin ring formation of osteoclasts. (A) F-actin ring expression in RAW264.7 transfected with mutant $\mathrm{LOX}_{\mathrm{G} 473 \mathrm{~A}}$ (MUT) plasmid and treated with 3-MA and RAPA detected by phalloidin staining. Magnification $\times 100$. (B) Semiquantitative analysis of the number of $\mathrm{F}$-actin ring by Image-Pro Plus software. ${ }^{* * *}, \mathrm{P}<0.0001$ vs. MUT represents significance.

promote the level of autophagy of osteoclast differentiation of RAW264.7. It could be inferred that $\operatorname{LOX}_{\mathrm{G} 473 \mathrm{~A}}$ interacted with autophagy to regulate the formation and absorption of osteoclasts.

Furthermore, osteoclast differentiation of RAW264.7 with mutant $\mathrm{LOX}_{\mathrm{G} 473 \mathrm{~A}}$ showed downregulated p-AMPK $\alpha$ protein expression and upregulated $\mathrm{p}$-mTOR protein expression after blocking of autophagy. Other research has shown that osteoprotegerin restrains osteoclast differentiation and bone resorption through regulating of autophagy via the AMPK/mTOR/p70S6K signaling pathway (35). AMPK is a key regulator of bone homeostasis by negatively regulating osteoclast formation and bone resorption (36). AMPK activation can also suppress the activation of mTOR, which can affect autophagy (37). It could be inferred that promoting autophagy would increase $\mathrm{LOX}_{\mathrm{G} 473 \mathrm{~A}}$-induced osteoclast differentiation by the p-AMPK $\alpha / \mathrm{mTOR}$ signal pathway. In addition, restricted mTOR activation also inhibits expression of CTSK and matrix metalloprotein-9, RANK and NFATC1 in osteoclasts (38), further indicating that the autophagy signaling pathway of $\mathrm{p}-\mathrm{AMPK} \alpha / \mathrm{mTOR}$ might inhibit the production of osteoclasts by restraining the NFATC1,
ACP5, and CTSK proteins as shown in our research. Considering the existence of other autophagic signaling pathways, such as PI3K/AKT/mTOR (39) and mTORindependent (40) signaling pathways, it is unclear whether they play a regulatory role in osteoclast formation, and we will follow up with further studies to explore whether LOXG473A regulates osteoclast formation through cross-talk of different autophagic pathways. Moreover, whether inhibiting osteoclast differentiation by suppressing autophagy brings other potential risks should be taken into consideration in subsequent studies.

\section{Conclusions}

In summary, $\operatorname{LOX}_{\mathrm{G} 473 \mathrm{~A}}$ could induce excessive activation of osteoclasts by enhancing activation of the AMPK $\alpha / \mathrm{mTOR}$ signaling pathway associated with autophagy. $\operatorname{LOX}_{\mathrm{G} 473 \mathrm{~A}}$ might be a pathogenic gene causing osteoporosis and could be a potential therapeutic target.

\section{Acknowledgments}

Funding: This work was supported by the National Natural 
A

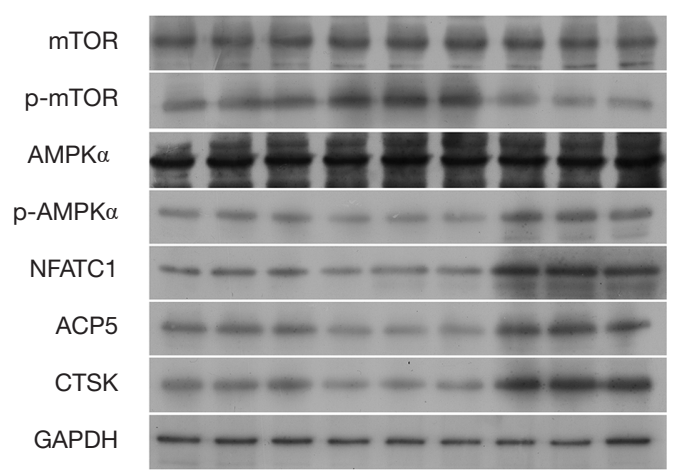

$289 \mathrm{kDa}$

$289 \mathrm{kDa}$

$62 \mathrm{kDa}$

$62 \mathrm{kDa}$

$90 \mathrm{kDa}$

$34 \mathrm{kDa}$

$45 \mathrm{kDa}$

$36 \mathrm{kDa}$
B p-mTOR/mTOR

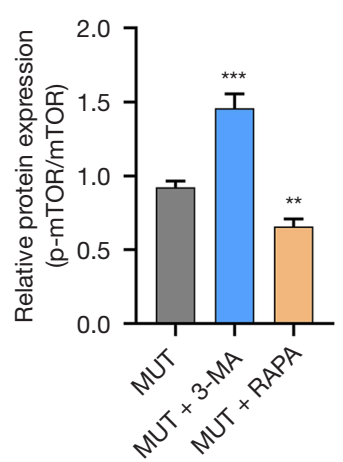

p-AMPK $\alpha / A M P K \alpha$

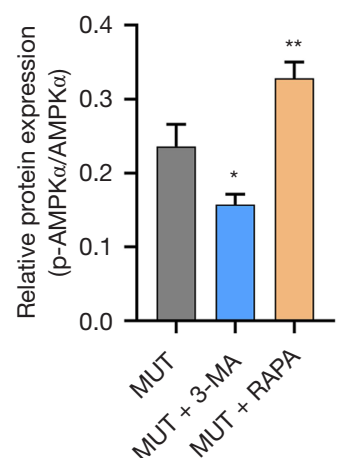

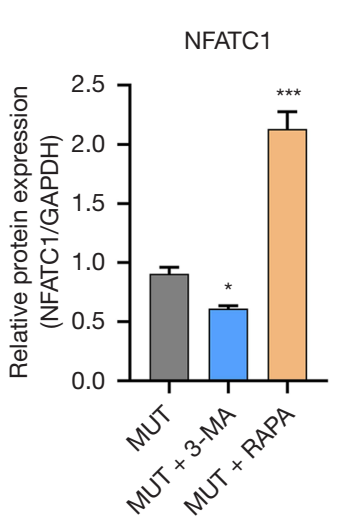
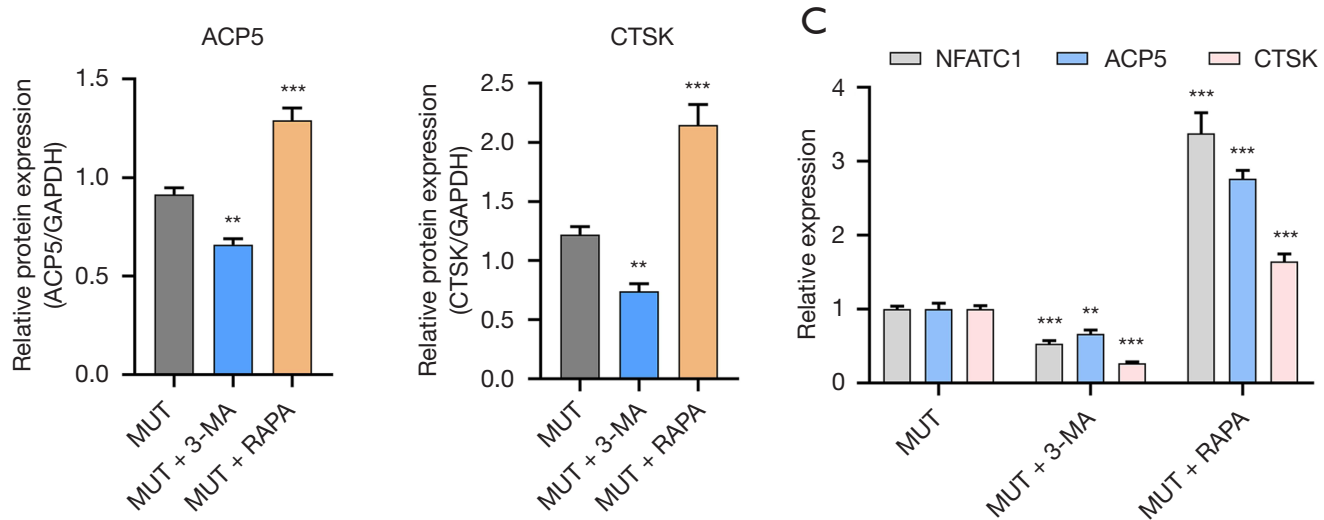

Figure 6 Effects of autophagy inhibitors and agonists on autophagy-related mechanism protein, as well as osteoclast protein and mRNA levels in osteoclasts. (A) Expression of autophagy-related mechanism protein and osteoclast protein in RAW264.7 transfected with mutant $\mathrm{LOX}_{\mathrm{G} 473 \mathrm{~A}}$ (MUT) plasmid and treated with 3-MA and RAPA detected by western blot. (B) Protein expression level in (A) was quantified by Image-Pro Plus software. (C) Osteoclast mRNA level in RAW264.7 transfected with MUT plasmid and treated with 3-MA and RAPA detected by qPCR. *, $\mathrm{P}<0.05$; **, $\mathrm{P}<0.01$; and ${ }^{\text {***}, ~} \mathrm{P}<0.001$ vs. MUT represent significant difference.

Science Foundation of China (NSFC, 81900802); Suzhou Youth Science and Education Project (KJXW2018014); Advantage discipline groups of the Second Affiliated Hospital of Soochow University (XKQ 2015001); The Project of State Key Laboratory of Radiation Medicine and Protection, Soochow University (GZK1202009).

\section{Footnote}

Reporting Checklist: The authors have completed the MDAR reporting checklist. Available at https://dx.doi. org/10.21037/atm-21-4474

Data Sharing Statement: Available at https://dx.doi. org/10.21037/atm-21-4474
Conflicts of Interest: All authors have completed the ICMJE uniform disclosure form (available at https://dx.doi. org/10.21037/atm-21-4474). The authors have no conflicts of interest to declare.

Ethical Statement: The authors are accountable for all aspects of the work in ensuring that questions related to the accuracy or integrity of any part of the work are appropriately investigated and resolved.

Open Access Statement: This is an Open Access article distributed in accordance with the Creative Commons Attribution-NonCommercial-NoDerivs 4.0 International License (CC BY-NC-ND 4.0), which permits the noncommercial replication and distribution of the article with 
the strict proviso that no changes or edits are made and the original work is properly cited (including links to both the formal publication through the relevant DOI and the license). See: https://creativecommons.org/licenses/by-nc-nd/4.0/.

\section{References}

1. Rossi M, Battafarano G, Pepe J, et al. The Endocrine Function of Osteocalcin Regulated by Bone Resorption: A Lesson from Reduced and Increased Bone Mass Diseases. Int J Mol Sci 2019;20:4502.

2. Kim M, Kim HS, Kim JH, et al. Chaenomelis fructus inhibits osteoclast differentiation by suppressing NFATc1 expression and prevents ovariectomy-induced osteoporosis. BMC Complement Med Ther 2020;20:35.

3. Song W, Xie J, Li J, et al. The Emerging Roles of Long Noncoding RNAs in Bone Homeostasis and Their Potential Application in Bone-Related Diseases. DNA Cell Biol 2020;39:926-37.

4. Li K, Chen S, Cai P, et al. MiRNA-483-5p is involved in the pathogenesis of osteoporosis by promoting osteoclast differentiation. Mol Cell Probes 2020;49:101479.

5. Yang TL, Shen H, Liu A, et al. A road map for understanding molecular and genetic determinants of osteoporosis. Nat Rev Endocrinol 2020;16:91-103.

6. Johnston CB, Dagar M. Osteoporosis in Older Adults. Med Clin North Am 2020;104:873-84.

7. Teitelbaum SL. Osteoclasts: what do they do and how do they do it? Am J Pathol 2007;170:427-35.

8. Anesi A, Generali L, Sandoni L, et al. From Osteoclast Differentiation to Osteonecrosis of the Jaw: Molecular and Clinical Insights. Int J Mol Sci 2019;20:4925.

9. Kim JH, Kim N. Signaling Pathways in Osteoclast Differentiation. Chonnam Med J 2016;52:12-7.

10. Okada K, Moon HJ, Finney J, et al. Extracellular Processing of Lysyl Oxidase-like 2 and Its Effect on Amine Oxidase Activity. Biochemistry 2018;57:6973-83.

11. Daley EJ, Pajevic PD, Roy S, et al. Impaired Gastric Hormone Regulation of Osteoblasts and Lysyl Oxidase Drives Bone Disease in Diabetes Mellitus. JBMR Plus 2019;3:e10212.

12. Cox TR, Rumney RMH, Schoof EM, et al. The hypoxic cancer secretome induces pre-metastatic bone lesions through lysyl oxidase. Nature 2015;522:106-10.

13. Cox TR, Erler JT, Rumney RMH. Established Models and New Paradigms for Hypoxia-Driven Cancer-Associated Bone Disease. Calcif Tissue Int 2018;102:163-73.

14. Fraga A, Ribeiro R, Coelho A, et al. Genetic polymorphisms in key hypoxia-regulated downstream molecules and phenotypic correlation in prostate cancer. BMC Urol 2017;17:12.

15. Wang G, Shen Y, Cheng G, et al. Lysyl Oxidase Gene G473A Polymorphism and Cigarette Smoking in Association with a High Risk of Lung and Colorectal Cancers in a North Chinese Population. Int J Environ Res Public Health 2016;13:635.

16. Gao X, Zhang S, Zhu Z. Lysyl oxidase rs 1800449 polymorphism and cancer risk among Asians: evidence from a meta-analysis and a case-control study of colorectal cancer. Mol Genet Genomics 2015;290:23-8.

17. Xiao W, He J, Fu W, et al. LOX gene polymorphisms are associated with osteoporotic vertebral compression fracture in postmenopausal Chinese women. Gene 2020;741:144543.

18. Guo XN, Ma X. The Effects of Propofol on Autophagy. DNA Cell Biol 2020;39:197-209.

19. Tan WSD, Shen HM, Wong WSF. Dysregulated autophagy in COPD: A pathogenic process to be deciphered. Pharmacol Res 2019;144:1-7.

20. Kim CJ, Shin SH, Kim BJ, et al. The Effects of Kaempferol-Inhibited Autophagy on Osteoclast Formation. Int J Mol Sci 2018;19:125.

21. Hocking LJ, Whitehouse C, Helfrich MH. Autophagy: a new player in skeletal maintenance? J Bone Miner Res 2012;27:1439-47.

22. AlQranei MS, Aljohani H, Majumdar S, et al. C-phycocyanin attenuates RANKL-induced osteoclastogenesis and bone resorption in vitro through inhibiting ROS levels, NFATc1 and NF- $\mathrm{\kappa B}$ activation. Sci Rep 2020;10:2513.

23. Hotokezaka H, Sakai E, Kanaoka K, et al. U0126 and PD98059, specific inhibitors of MEK, accelerate differentiation of RAW264.7 cells into osteoclast-like cells. J Biol Chem 2002;277:47366-72.

24. Chen X, Zhi X, Pan P, et al. Matrine prevents bone loss in ovariectomized mice by inhibiting RANKL-induced osteoclastogenesis. FASEB J 2017;31:4855-65.

25. Colombo C, Li M, Watanabe S, et al. Polymer Nanoparticle Engineering for Podocyte Repair: From in Vitro Models to New Nanotherapeutics in Kidney Diseases. ACS Omega 2017;2:599-610.

26. Li J, Zeng L, Xie J, et al. Inhibition of Osteoclastogenesis and Bone Resorption in vitro and in vivo by a prenylflavonoid xanthohumol from hops. Sci Rep 2015;5:17605.

27. Nara Y, Kitaura H, Ogawa S, et al. Anti-c-fms Antibody 
Prevents Osteoclast Formation and Bone Resorption in Co-Culture of Osteoblasts and Osteoclast Precursors In Vitro and in Ovariectomized Mice. Int J Mol Sci 2020;21:6120.

28. Li W, Zhu HM, Xu HD, et al. CRNDE impacts the proliferation of osteoclast by estrogen deficiency in postmenopausal osteoporosis. Eur Rev Med Pharmacol Sci 2018;22:5815-21.

29. Hasegawa T, Kikuta J, Sudo T, et al. Identification of a novel arthritis-associated osteoclast precursor macrophage regulated by FoxM1. Nat Immunol 2019;20:1631-43.

30. Huang J, Lin D, Wei Z, et al. Parathyroid Hormone Derivative with Reduced Osteoclastic Activity Promoted Bone Regeneration via Synergistic Bone Remodeling and Angiogenesis. Small 2020;16:e1905876.

31. Saad FA. Novel insights into the complex architecture of osteoporosis molecular genetics. Ann N Y Acad Sci 2020;1462:37-52.

32. Reynaud C, Ferreras L, Di Mauro P, et al. Lysyl Oxidase Is a Strong Determinant of Tumor Cell Colonization in Bone. Cancer Res 2017;77:268-78.

33. Pichu S, Sathiyamoorthy J, Vimalraj S, et al. Impact of lysyl oxidase (G473A) polymorphism on diabetic foot ulcers. Int J Biol Macromol 2017;103:242-7.

34. Song L, Tan J, Wang Z, et al. Interleukin-17A facilitates osteoclast differentiation and bone resorption via activation of autophagy in mouse bone marrow macrophages. Mol

Cite this article as: Zhang B, Luo C, Xiao W. Induction of osteoclast formation by LOX mutant (LOXG473A) through regulation of autophagy. Ann Transl Med 2021;9(18):1474. doi: 10.21037/atm-21-4474
Med Rep 2019;19:4743-52.

35. Tong X, Gu J, Song R, et al. Osteoprotegerin inhibit osteoclast differentiation and bone resorption by enhancing autophagy via AMPK/mTOR/p70S6K signaling pathway in vitro. J Cell Biochem 2018. [Epub ahead of print]. doi: 10.1002/jcb.27468.

36. Kang H, Viollet B, Wu D. Genetic deletion of catalytic subunits of AMP-activated protein kinase increases osteoclasts and reduces bone mass in young adult mice. $\mathrm{J}$ Biol Chem 2013;288:12187-96.

37. Wang Q, Wei S, Zhou S, et al. Hyperglycemia aggravates acute liver injury by promoting liver-resident macrophage NLRP3 inflammasome activation via the inhibition of AMPK/mTOR-mediated autophagy induction. Immunol Cell Biol 2020;98:54-66.

38. Tong X, Zhang C, Wang D, et al. Suppression of AMPactivated protein kinase reverses osteoprotegerin-induced inhibition of osteoclast differentiation by reducing autophagy. Cell Prolif 2020;53:e12714.

39. Xu Z, Han X, Ou D, et al. Targeting PI3K/AKT/mTORmediated autophagy for tumor therapy. Appl Microbiol Biotechnol 2020;104:575-87.

40. Williams A, Sarkar S, Cuddon P, et al. Novel targets for Huntington's disease in an mTOR-independent autophagy pathway. Nat Chem Biol 2008;4:295-305.

(English Language Editor: K. Brown) 